\title{
Rosa e Azul: Sexo e Idade no Teste de Pfister
}

\author{
Anna Elisa Villemor-Amaral - Universidade São Francisco, Itatiba, Brasil \\ Fabiola Cristina Biasi - Universidade São Francisco, Itatiba, Brasil \\ Lucila Moraes Cardoso - Universidade Estadual do Ceará, Fortaleza, Brasil \\ Pâmela Malio Pardini Pavan - Universidade São Francisco, Itatiba, Brasil \\ Raquel Rossi Tavella - Centro Universitário Nossa Senhora do Patrocínio, Itu, Brasil
}

\begin{abstract}
Resumo
Como um dos indicadores de dinâmica emocional no Teste das Pirâmides Coloridas de Pfister trata da seleção de cores, considera-se importante verificar se a idade ou o sexo tem implicações na escolha das cores, sob influência de aspectos culturais. Dentre as possibilidades, enfatiza-se a ideia prevalente na cultura ocidental de que o rosa seria a cor das meninas e o azul seria a cor dos meninos. Para tal, comparou-se o uso das diferentes tonalidades de azul e vermelho no Pfister de 734 crianças e adultos de ambos os sexos. As participantes do sexo feminino demonstraram preferência por tons mais claros, culturalmente associados à feminilidade, e os participantes do sexo masculino, por tons mais escuros, sugerindo controle emocional. Observou-se também aumento do uso de tonalidades mais claras por meninos quando comparados com homens e de tonalidades mais escuras por meninas em relação às mulheres. Conclui-se que a escolha das cores, especialmente azul e rosa, difere conforme o sexo e a idade, de acordo com certas influências culturais.

Palavras-chaves: avaliação psicológica, teste das pirâmides coloridas, sexo
\end{abstract}

Pink and Blue: Sex and Age in the Pfister's Test

\begin{abstract}
As one of the indicators of emotional dynamics in the Color Pyramid Test of Pfister is related to the color choices, it is important to check whether age or gender are related to the choice of colors, under the influence of cultural factors. Among the possibilities, this work emphasizes the prevalent idea in the occidental culture that pink was the color for girls and blue would be the color of the boys. To achieve this goal, we compared the use of different shades of blue and red in Pfister tests of 734 children and adults of both genders. The female participants showed a preference for lighter shades, which is socially attributed to femininity, and male participants in darker colors, suggesting emotional control. It was also observed increased lighter shades among boys when compared to men and darker shades for girls in relation to women. It suggests that the choice of the colors differ among sex and age, specially the pink and blue, according some cultural influences.

Keywords: psychological assessment, color pyramid test, gender
\end{abstract}

Rosado y Azul: Sexo y Edad en el Test de Pfister

\section{Resumen}

La selección de los colores es uno de los indicadores de dinámica emocional en el Test de Las Pirámides Coloridas de Pfister y se considera importante verificar si la edad y el sexo, bajo la influencia de aspectos culturales, influyen en la elección de los mismos. Entre las posibilidades, se hace hincapié en la idea prevaleciente en la cultura occidental de que rosado sería color de niñas y azul de niños. Por lo tanto, se comparó el uso de diferentes tonos de azul y rojo en el Pfister entre 734 niños y adultos de ambos sexos. Las participantes femeninas mostraron una preferencia por tonos más claros, culturalmente asociado a femineidad, y los participantes masculinos por tonos más oscuros, sugiriendo control emocional. También se observó un mayor uso de tonos más claros por niños, en comparación con los hombres; y tonos más oscuros por niñas, en relación con las mujeres. De ello se desprende que la elección de colores, especialmente azul y rosado, difiere según el sexo y la edad, de acuerdo con ciertas influencias culturales.

Palabras claves: evaluación psicológica, test de las pirámides coloridas, sexo

\section{Introdução}

O Teste das Pirâmides Coloridas de Pfister (TPC) é um método expressivo de avaliação psicológica, que favorece a exteriorização de conteúdos internos e permite a investigação de aspectos emocionais da dinâmica de personalidade da pessoa avaliada (Guntert, 2000; Peres, Santos, Rodrigues \& Okino, 2007). Esse tipo de método favorece a manifestação de conteúdos afetivos e cognitivos que podem não estar aparentes nos comportamentos manifestos do indivíduo no momento da avaliação (Franco, 2009).

Esse método foi criado pelo Suíço Max Pfister em 1952. No histórico sobre o autor, há relatos sobre seu gosto por artes desde a juventude, interesse pelas cores e a formação em Arquitetura (Villemor-Amaral, 2012). A ideia de construir pirâmides a partir do jogo de quadrículos coloridos parece ter sido mais influenciada 
pelas vivências do autor no campo das artes do que por experiências científicas naquele tempo.

O caráter científico do instrumento foi alcançado pelos psicólogos Robert Heiss e Hildegard Hiltmann da Universidade de Friburgo, na Alemanha, anos após a morte de Pfister (Villemor-Amaral, 2012). Esses autores assumiram a tarefa de investigar, sistematizar e divulgar o TPC enquanto instrumento de avaliação psicológica, tendo proposto algumas modificações no método original, tal como sugerir o uso de três pirâmides ao invés de apenas uma, como era recomendado inicialmente (Marques, 1988).

Nos últimos anos, houve um aumento na frequência de estudos científicos com o TPC no Brasil. Numa revisão da literatura nacional sobre o TPC, Silva e Cardoso (2012) encontraram 12 artigos completos publicados na Biblioteca Virtual de Saúde em Psicologia (BVS-Psi) entre 2001 e 2011. Após realizar os mesmos procedimentos usados por essas pesquisadoras, considerando agora o período entre 2012 e 2014, foram obtidos oito novos artigos. Desse total de 20 artigos, 12 (60\%) tinham como objetivo estudar as qualidades psicométricas do instrumento. $\mathrm{O}$ conjunto de trabalhos publicados permite afirmar que o TPC tem estudos normativos para uso com crianças (Villemor-Amaral, Farah, \& Primi, 2014a), com adolescentes (Pasian, Barroso, \& Theodozio, 2014), com adultos (Villemor-Amaral, Primi, Farah, Cardoso, \& Franco, 2003; Villemor-Amaral, Pianowski, \& Goncalves, 2008) e com idosos (Bastos-Formighieri \& Pasian, 2012), além dos estudos de precisão (Farah, Cardoso, \& Villemor-Amaral, 2014; Villemor-Amaral, Cardoso, Pavan, Tavella, \& Biasi, 2014b) e de evidências de validade para uso com crianças (Farah et al., 2014; Villemor-Amaral, Pardini, Tavella, Biasi, \& Migoranci, 2012; Villemor-Amaral \& Quirino, 2013) e para o diagnóstico diferencial de psicopatologias.

Nas investigações para diagnóstico diferencial, os grupos não clínicos foram compostos por pessoas sem histórico de busca de ajuda psicológica e psiquiátrica e que, nas entrevistas que antecediam a aplicação do teste, não aparentassem ter demanda para tal, e o grupo clínico formado por pessoas com quadros psicopatológicos já diagnosticados e confirmados pela entrevista clínica semiestruturada baseada no DSM-IV (SCID). Na realização desses estudos, levantaram-se as hipóteses sobre quais indicadores teoricamente seriam sensíveis para diferenciar os grupos psicopatológicos e, após análise dos resultados, confirmou-se que combinações específicas do aspecto formal, da porcentagem das cores e da fórmula cromática podem ser importantes para o diagnóstico complementar do transtorno esquizofrênico (Villemor-Amaral et al., 2005), transtorno de pânico (Villemor-Amaral et al., 2004), transtorno depressivo (Villemor-Amaral et al., 2004), transtorno alcoolista (Villemor-Amaral, Silva, \& Primi, 2003) e transtorno obsessivo compulsivo (Villemor-Amaral, Silva, \& Primi, 2002).

A título de exemplo, pode-se citar a cor vermelha, que, no teste de Pfister, associa-se "à extroversão, à irritabilidade, à impulsividade e à agressividade" (Villemor-Amaral, 2012, p. 81), que apareceu aumentada nos grupos de pacientes esquizofrênicos e também no grupo de alcoolistas, grupos esses que, pela própria psicopatologia, possuem características mais associadas à impulsividade e à perda do controle da agressividade. Já a cor azul, que está comumente relacionada, dependendo da quantidade e tonalidade, à capacidade de controle e adaptação, apareceu aumentada no transtorno do pânico que se associa mais a características associadas à necessidade de controle, assim como o marrom, significativamente aumentado em pacientes com Transtorno Obsessivo Compulsivo.

O TPC costuma ser bem aceito pelas pessoas que o realizam, tendo um caráter lúdico na medida em que a tarefa do examinando consiste em preencher esquemas de pirâmides com quadrículos coloridos. $\mathrm{O}$ azul, o vermelho e o verde são as cores mais frequentemente usadas no teste (Villemor-Amaral, 2012), estando disponíveis em quatro tonalidades, em que as tonalidades 1 são as mais claras e gradativamente vão ficando mais escuras até a tonalidade 4, com maior impregnação de preto. Nessa graduação das cores, destaca-se que o vermelho 1, por ser o tom mais impregnado de branco, costuma ser considerado pelos examinandos como "rosa". Dentro desse espectro, todas as tonalidades designadas com o número 1 sugerem características emocionais mais associadas à fragilidade, dada a diluição da densidade da cor por meio do branco, e as tonalidades mais escuras se associam frequentemente à necessidade de controle e repressão devido à impregnação do preto que escurece e turva a vivacidade da cor (Villemor Amaral, 1978).

Com frequência, os examinandos comentam, enquanto escolhem as cores para construção das pirâmides, influências decorrentes de preferências pessoais ou claramente relacionadas com aspectos culturais, falando das cores que mais gostam ou eventualmente mencionando o significado culturalmente atribuído a cada cor. Quanto a esse aspecto, não há consenso 
sobre os fatores envolvidos na escolha por determinadas cores, sendo que alguns pesquisadores defendem que a preferência por cores se dá por fatores biológicos (LeDoux, 1998; Hurlbert \& Ling, 2007), enquanto outros argumentam a favor de influência dos aspectos culturais (LoBue \& DeLoach, 2011; Milne \& Greenway, 1999).

Para LeDoux (1998), ainda que as pessoas estabeleçam associações com as suas vivências e aspectos difundidos no senso comum, diversos estudos têm apontado outros fatores que parecem subsidiar a escolha por cores de um modo geral, trazendo aportes não só relacionados com as propriedades físicas da luz, mas integrando-os aos avanços das neurociências na compreensão da relação entre cor e emoção. Essas influências parecem, portanto, derivar principalmente da física e, mais recentemente, da neurociência. Os primeiros estudos da cor enquanto fenômeno físico são atribuídos a Isaac Newton que, em 1672, concluiu que as cores são resultado da decomposição do raio luminoso. Essa teoria, posteriormente, possibilitou compreender que, ao perceber as cores, as pessoas estão na realidade sendo estimuladas por determinadas ondas eletromagnéticas, constituindo-se um estímulo que atinge os receptores visuais com intensidades distintas.

Hurlbert e Ling (2007) afirmam que, embora haja abundante evidência para as diferenças de sexo em outros domínios visuais, tal como percepção da cor, não existe evidência conclusiva em relação a possíveis diferenças sexuais na preferência de cor. Para os pesquisadores, as diferenças nas escolhas das cores se relacionam com as dimensões neurais que fundamentam a codificação de cores no sistema visual humano. A diferença entre os sexos, nesse caso, consiste numa sensibilização na evolução de comportamentos específicos relacionados ao sexo, aos ancestrais, como, por exemplo, o fato de que as mulheres discriminaram antes a cor vermelha, pois tinham que identificar o fruto maduro em contraste com a folhagem verde, enquanto para os homens, caçadores, a identificação do movimento da caça era mais fundamental. Essas concepções estão de acordo com as teorias sobre a especialização hemisférica cerebral e as diferenças entre os sexos (Yazigi, 1995)

Ainda Hulbert e Ling (2007), visando verificar suas hipóteses, realizaram um estudo no qual participaram 208 pessoas com idades entre 20 e 26 anos, sendo 171 britânicos caucasianos, 79 do sexo masculino, e 37 chineses, 19 do sexo masculino, que moravam no Reino Unido por no máximo 3 anos. Após serem expostos a três experiências diferentes, os resultados indicaram que as mulheres preferiram a cor vermelho-púrpura e os homens a cor azul ou verde, embora de modo menos acentuado, uma vez que as mulheres também revelaram preferência por essas cores. $\mathrm{O}$ estudo também revelou que os chineses deram mais preferência ao vermelho que os britânicos, potencialmente pela significação cultural do vermelho como sendo a cor da boa sorte na China.

LoBue e DeLoache (2011) afirmaram também que a preferência e utilização das cores pode estar associada com aspectos culturais e exemplificam sua hipótese com o fato dos bebês meninos, quando nascem, serem vestidos de azul e as meninas de rosa. Alertam que as propagandas e a mídia em geral também enfatizam as cores rosa nas roupas, sapatos, brinquedos, etc., das meninas e azul para os meninos. Esses pesquisadores realizaram um estudo com o objetivo de verificar a preferência das cores de crianças do sexo masculino e feminino. Participaram do estudo 192 crianças de ambos os sexos, que foram divididas em seis grupos de 32 crianças de acordo com a idade, que variou de 7 meses a 5 anos. Foram oferecidos oito pares de objetos para as crianças e foi pedido para que elas escolhessem um. Em cada par, um dos objetos era sempre rosa e o outro tinha cores que variavam entre verde, azul, amarelo e laranja. Observou-se que crianças com menos de 2 anos não diferiram na frequência com as que escolheram objetos cor de rosa. Mas a partir de 2 anos de idade, a escolha de objetos cor de rosa foi significativamente mais frequente nas meninas do que nos meninos, sendo que até os 3 anos as meninas demonstraram uma preferência significativa para o rosa sobre outras cores. Além disso, entre as idades de 3 e 4, os meninos mostram uma anulação no interesse pela cor rosa. Esses resultados revelaram que as diferenças sexuais na preferência e atração da cor rosa por meninas jovens e uma crescente rejeição do rosa por meninos.

Milne e Greenway (1999) testaram a hipótese de que os meninos e as meninas diferem na utilização de cor em desenhos. Participaram da pesquisa 61 pacientes ambulatoriais de saúde mental, de ambos os sexos, com idades entre 4 e 14 anos (Média $=8,52$ e $D P=2,23$ ). Os sujeitos foram divididos em três grupos, considerando-se a idade. O primeiro grupo foi composto por indivíduos com menos de 6,56 anos, o segundo com idades entre 6,56 e 10,63 e o terceiro grupo tinha idade maior que 10,63 anos. Foi solicitado inicialmente que a criança desenhasse algo de sua livre escolha, posteriormente foi pedido que desenhasse a si mesma, uma casa, uma árvore e uma pessoa, totalizando cinco desenhos. 
Variou-se a ordem de solicitação dos quatro últimos desenhos. Os resultados indicaram que os meninos mais jovens usaram cor com maior frequência do que meninos mais velhos e que as meninas usam cor independentemente da idade.

Considerando que são escassas as pesquisas científicas sobre o significado das cores e tonalidades e sobre a preferência cromática em função da idade, do sexo ou características emocionais específicas, incluindo as patológicas, o presente estudo teve como objetivo verificar se o uso das cores azul e vermelho, bem como suas tonalidades, no Teste das Pirâmides Coloridas de Pfister, diferem de acordo com o sexo e a faixa etária.

\section{Método}

\section{Participantes/Banco de Dados}

Para realização da presente pesquisa foi utilizado o banco de dados usado para compor as amostras normativas do manual do teste de Pfister para adultos (Villermor-Amaral, 2012) e para crianças (Villemor-Amaral, 2014), totalizando 734 protocolos administrados em cidades do interior paulista e mineiro. A partir do banco de dados, obteve-se os protocolos de 206 adultos com idades entre 19 a 78 anos, sendo 117 do sexo masculino e 89 do sexo feminino, e de 528 crianças com idades entre 6 e 12 anos, sendo 219 meninos e 309 meninas.

\section{Instrumentos}

Teste das Pirâmides Coloridas de Pfister (Villemor-Amaral, 2012)

No teste de Pfister, solicita-se ao examinando que execute três pirâmides que fiquem do seu gosto, construindo-as uma de cada vez. Ao final da execução das três pirâmides é realizado o inquérito, indagando-se ao examinando qual pirâmide ele mais gostou e o porquê, e a que menos gostou e o porquê. $\mathrm{O}$ tempo estimado para a aplicação é de 30 minutos. O material consiste em três cartões em papel bege, com o esquema de uma pirâmide cada, um conjunto de quadrículos coloridos, compostos por 10 cores que estão divididas em 24 tonalidades.

\section{Procedimentos}

\section{Coleta de Cados}

Utilizaram-se os protocolos armazenados nos bancos de dados para composição das amostras normativas do TPC tanto para adultos quanto para crianças (Villemor-Amaral, 2012, 2014). Destaca-se que ambos os bancos foram compostos pelo mesmo laboratório de pesquisa, assegurando a uniformidade dos procedimentos de coleta de dados. Em todos os casos o TPC foi administrado em sessão individual de aproximadamente 30 minutos.

Para minimizar possíveis erros de codificação, todos os protocolos foram avaliados pelas examinadoras e revisados pela coordenadora da pesquisa. Além disso, em cada um dos estudos foi verificada a fidedignidade entre avaliadores, verificando-se índices de concordância superiores a $86 \%$ (Villemor-Amaral, 2012, 2014).

\section{Análise dos Dados}

Após os dois bancos terem sido unificados, verificou-se a composição da amostra considerando-se o sexo e a idade dos participantes. Posteriormente foi utilizado o teste estatístico t de Student visando a comparação do uso das diferentes tonalidades de azul e vermelho em função do sexo, masculino e feminino, e posteriormente em função dos agrupamentos por idade, crianças e adultos. Além disso, foi calculado o $d$ de Cohen para analisar o efeito da magnitude, considerando-se $d=$ 0,20 como um efeito de pequena magnitude, $d=0,50$ uma magnitude intermediária e $d=0,80$ uma grande magnitude (Cohen, 1992).

\section{Considerações Éticas}

Destaca-se que a presente pesquisa contou com o uso de dois bancos de dados, um usado para compor a amostra normativa de adultos e o outro a amostra normativa de crianças. Ambas as pesquisas foram aprovadas pelo Comitê de Ética em Pesquisa da Universidade São Francisco.

\section{Resultados}

Para verificar se há diferenças na escolha das cores de acordo com o sexo e idade, primeiramente foi feita a comparação das médias das cores azul e vermelho entre homens e mulheres adultos. Observa-se, na Tabela 1, que as mulheres apresentaram médias significativamente maiores nos tons $\mathrm{Az} 2 \mathrm{e} \mathrm{Vm} 1$, enquanto que os homens tiveram aumento significativo em Az4 e Vm4.

Dando continuidade às comparações por sexo, na Tabela 2, verifica-se o resultado da comparação entre meninos e meninas. As meninas apresentaram aumento 
significativo de Az1 e Vm1 e os meninos o aumento do Az4 e Vm2. Com relação à magnitude do efeito Az1, $\mathrm{Az} 4$ e Vm2 tiveram uma magnitude pequena, enquanto que Vm1 revela magnitude intermediária de acordo com os padrões de Cohen (1992).

Após fazer as análises por sexo, foi feita a comparação entre crianças e adultos. Assim, na Tabela 3, é possível visualizar os dados sobre o uso das tonalidades vermelho e azul do TPC entre mulheres e meninas, verificando-se diferenças estaticamente significativas entre os grupos. As mulheres usaram mais as cores Az2 e Az3, enquanto as meninas tiveram uma frequência mais elevada de uso do Az4 e Vm4. As magnitudes da diferença de Az3 e Vm4 foram pequenas e as de Az2 e Az4 foram intermediárias.

Por fim, foi feita a comparação entre meninos e homens, conforme dados expostos na Tabela 4. Nota-se o aumento de Vm1 no grupo dos meninos em comparação com o grupo de homens. A magnitude de efeito foi considerada pequena.

\section{Discussão}

O TPC é um instrumento que vem acumulando estudos de precisão (Farah et al., 2014 e Villemor-Amaral et al., 2014b) e de evidências de validade. Tratando-se de um instrumento interessante na medida em que pode ser usado com pessoas de diferentes idades, incluindo-se a indicação de uso com crianças (Farah et al., 2014; Villemor-Amaral et al., 2012; Villemor-Amaral \& Quirino, 2013), e com pacientes psiquiátricos (Villemor-Amaral et al., 2005; Villemor-Amaral et al., 2004; Villemor-Amaral et al., 2003 e Villemor-Amaral et al., 2002).

A frequência de uso das cores, no TPC, é um dos principais indicadores para avaliar a dinâmica emocional do examinando (Villemor-Amaral, 2012; 2014), requisitando atenção à sua interpretação. A diversidade cultural brasileira, associada à suposição de que as diferenças culturais levariam a diferenças na escolha das cores, justificou o estudo de Villemor-Amaral et al. (2008), que compararam uma amostra do nordeste com o sudeste brasileiro, sem encontrar diferenças estatisticamente significativas entre as amostra das duas regiões. De modo semelhante, a preocupação sobre a possível existência de influências culturais sobre a escolha das cores, o presente estudo buscou verificar se isso difere conforme o sexo e a idade, seguindo padrões culturalmente estabelecidos.

Ao analisar as Tabelas 2 e 3 do presente estudo, observa-se que as mulheres apresentaram aumento de

Tabela 1

Comparação das Médias das Tonalidades por Sexo dos Adultos

\begin{tabular}{|c|c|c|c|c|c|c|}
\hline Tons & Sexo & $M$ & $D P$ & $t$ & $d$ & $p$ \\
\hline \multirow[t]{2}{*}{$\mathrm{Az} 1$} & masculino & 1,85 & 2,53 & $-1,01$ & 0,14 & 0,311 \\
\hline & feminino & 2,20 & 2,44 & & & \\
\hline \multirow[t]{2}{*}{$\mathrm{Az} 2$} & masculino & 1,64 & 1,77 & $-2,83$ & 0,40 & 0,010 \\
\hline & feminino & 2,40 & 2,09 & & & \\
\hline \multirow[t]{2}{*}{ Az3 } & masculino & 2,09 & 2,76 & $-0,80$ & 0,13 & 0,936 \\
\hline & feminino & 2,11 & 1,75 & & & \\
\hline \multirow[t]{2}{*}{$\mathrm{Az} 4$} & masculino & 2,01 & 2,22 & 4,17 & 0,55 & $<0,001$ \\
\hline & feminino & 1,00 & 1,21 & & & \\
\hline \multirow[t]{2}{*}{ Vm1 } & masculino & 1,51 & 1,67 & $-5,27$ & 0,75 & $<0,001$ \\
\hline & feminino & 3,06 & 2,52 & & & \\
\hline \multirow[t]{2}{*}{$\mathrm{Vm} 2$} & masculino & 2,97 & 3,08 & 0,50 & $-0,07$ & 0,619 \\
\hline & feminino & 2,78 & 2,48 & & & \\
\hline \multirow[t]{2}{*}{$\mathrm{Vm} 3$} & masculino & 1,17 & 1,61 & 0,96 & $-0,13$ & 0,337 \\
\hline & feminino & 0,97 & 1,36 & & & \\
\hline \multirow[t]{2}{*}{$\mathrm{Vm} 4$} & masculino & 0,80 & 1,31 & 1,95 & 0,28 & 0,050 \\
\hline & feminino & 0,49 & 0,81 & & & \\
\hline
\end{tabular}


Tabela 2

Comparação das Médias das Tonalidades por Sexo das Crianças

\begin{tabular}{|c|c|c|c|c|c|c|}
\hline Tons & Sexo & M & $D P$ & $t$ & $d$ & $p$ \\
\hline \multirow[t]{2}{*}{ Az1 } & masculino & 1,87 & 1,42 & $-2,14$ & 0,19 & 0,030 \\
\hline & feminino & 2,21 & 2,07 & & & \\
\hline \multirow[t]{2}{*}{$\mathrm{Az} 2$} & masculino & 1,67 & 1,48 & 0,75 & $-0,07$ & 0,451 \\
\hline & feminino & 1,57 & 1,39 & & & \\
\hline \multirow[t]{2}{*}{$\mathrm{Az} 3$} & masculino & 1,80 & 1,64 & 0,765 & $-0,06$ & 0,445 \\
\hline & feminino & 1,70 & 1,59 & & & \\
\hline \multirow[t]{2}{*}{$\mathrm{Az} 4$} & masculino & 2,08 & 1,64 & 2,48 & 0,22 & 0,010 \\
\hline & feminino & 1,75 & 1,35 & & & \\
\hline \multirow[t]{2}{*}{$\mathrm{Vm} 1$} & masculino & 1,90 & 1,47 & $-7,40$ & 0,65 & $<0,001$ \\
\hline & feminino & 3,15 & 2,17 & & & \\
\hline \multirow[t]{2}{*}{$\mathrm{Vm} 2$} & masculino & 2,82 & 2,25 & 2,19 & 0,20 & 0,030 \\
\hline & feminino & 2,43 & 1,64 & & & \\
\hline \multirow[t]{2}{*}{$\mathrm{Vm} 3$} & masculino & 1,38 & 1,31 & 1,32 & $-0,11$ & 0,187 \\
\hline & feminino & 1,24 & 1,20 & & & \\
\hline \multirow[t]{2}{*}{$\mathrm{Vm} 4$} & masculino & 0,90 & 1,26 & 1,15 & $-0,10$ & 0,251 \\
\hline & feminino & 0,79 & 1,03 & & & \\
\hline
\end{tabular}

Tabela 3

Comparação das Médias das Tonalidades entre Mulheres e Meninas

\begin{tabular}{|c|c|c|c|c|c|c|}
\hline Tons & Sexo & M & $D P$ & $t$ & $d$ & $p$ \\
\hline \multirow[t]{2}{*}{ Az1 } & mulheres & 2,20 & 2,44 & $-0,45$ & 0,00 & 0,956 \\
\hline & meninas & 2,21 & 2,07 & & & \\
\hline \multirow[t]{2}{*}{$\mathrm{Az} 2$} & mulheres & 2,38 & 2,10 & 3,42 & 0,51 & $<0,001$ \\
\hline & meninas & 1,57 & 1,40 & & & \\
\hline \multirow[t]{2}{*}{$\mathrm{Az} 3$} & mulheres & 2,21 & 1,93 & 2,31 & 0,31 & 0,020 \\
\hline & meninas & 1,70 & 1,59 & & & \\
\hline \multirow[t]{2}{*}{$\mathrm{Az} 4$} & mulheres & 0,99 & 1,21 & $-4,77$ & 0,58 & $<0,001$ \\
\hline & meninas & 1,75 & 1,35 & & & \\
\hline \multirow[t]{2}{*}{ Vm1 } & mulheres & 3,06 & 2,52 & $-0,35$ & 0,00 & 0,723 \\
\hline & meninas & 3,15 & 2,16 & & & \\
\hline \multirow[t]{2}{*}{$\mathrm{Vm} 2$} & mulheres & 2,78 & 2,48 & 1,55 & $-0,15$ & 0,120 \\
\hline & meninas & 2,43 & 1,63 & & & \\
\hline \multirow[t]{2}{*}{$\mathrm{Vm} 3$} & mulheres & 0,97 & 1,37 & $-1,81$ & 0,20 & 0,071 \\
\hline & meninas & 1,24 & 1,20 & & & \\
\hline \multirow[t]{2}{*}{$\mathrm{Vm} 4$} & mulheres & 0,49 & 0,81 & $-2,83$ & 0,30 & 0,010 \\
\hline & meninas & 0,79 & 1,03 & & & \\
\hline
\end{tabular}


Tabela 4

Comparação das Médias das Tonalidades entre Homens e Meninos

\begin{tabular}{|c|c|c|c|c|c|c|}
\hline Tons & Grupo & $M$ & $D P$ & $t$ & $d$ & $p$ \\
\hline \multirow[t]{2}{*}{$\mathrm{Az} 1$} & homens & 1,85 & 2,53 & $-0,06$ & 0,01 & 0,952 \\
\hline & meninos & 1,86 & 1,41 & & & \\
\hline \multirow[t]{2}{*}{$\mathrm{Az} 2$} & homens & 1,64 & 1,77 & $-0,14$ & 0,02 & 0,881 \\
\hline & meninos & 1,67 & 1,48 & & & \\
\hline \multirow[t]{2}{*}{ Az3 } & homens & 2,09 & 2,76 & 1,17 & $-0,13$ & 0,243 \\
\hline & meninos & 1,80 & 1,64 & & & \\
\hline \multirow[t]{2}{*}{$\mathrm{Az} 4$} & homens & 2,01 & 2,22 & $-0,32$ & 0,03 & 0,747 \\
\hline & meninos & 2,08 & 1,63 & & & \\
\hline \multirow[t]{2}{*}{ Vm1 } & homens & 1,51 & 1,67 & $-2,21$ & 0,25 & 0,030 \\
\hline & meninos & 1,90 & 1,47 & & & \\
\hline \multirow[t]{2}{*}{$\mathrm{Vm} 2$} & homens & 2,97 & 3,08 & 0,53 & $-0,05$ & 0,595 \\
\hline & meninos & 2,82 & 2,24 & & & \\
\hline \multirow[t]{2}{*}{$\mathrm{Vm} 3$} & homens & 1,17 & 1,61 & $-1,29$ & 0,14 & 0,196 \\
\hline & meninos & 1,38 & 1,31 & & & \\
\hline \multirow[t]{2}{*}{$\mathrm{Vm} 4$} & homens & 0,80 & 1,31 & $-0,69$ & 0,08 & 0,491 \\
\hline & meninos & 0,90 & 1,26 & & & \\
\hline
\end{tabular}

Vm1. Esses dados corroboram com LoBue e DeLoache (2011) que relataram que as escolhas de brinquedos e outros objetos rosa é mais frequente em meninas do que em meninos, sendo que, na presente pesquisa, a diferença também apareceu na comparação entre mulheres e homens adultos. Villemor-Amaral (2014) aponta que essas diferenças podem expressar tendências culturais, uma vez que o rosa é uma cor habitualmente ligada à feminilidade na sociedade contemporânea ocidental. A esse resultado soma-se o fato de que homens apresentaram menos Vm1 do que meninos (Tabela 5). A diminuição de uso do $\mathrm{Vm} 1$ da infância à fase adulta pelo sexo masculino pode se dar por fatores culturais, no sentido de usarem a observação do meio em que vivem para determinar a preferência pelas cores, respeitando um padrão cultural, conforme apontado por LoBue e DeLoache (2011).

Segundo Heller (2011), foi apenas após a era Vitoriana que o rosa passou a ser a cor das meninas em oposição ao azul como cor dos meninos na cultura ocidental. Por outro lado, não se pode esquecer que não é sem razões físicas ou fisiológicas que essas cores vêm sendo associadas com infância ou feminilidade, uma vez que são tons mais delicados e suaves, atributos compatíveis com os valores culturalmente esperados para crianças e mulheres. Em outras palavras, a escolha de cores associa-se com valores culturais, mas os valores culturais são, em certa medida, determinados pelas propriedades mais ou menos estimulantes das cores, conforme apontado por LeBoux (1998) e Hurlbert e Ling (2007).

É interessante notar também o aumento do Az4 tanto nos adultos quanto nas crianças do sexo masculino (Tabelas 2 e 3). As tonalidades mais escuras, no TPC estão relacionadas a uma necessidade de controle de caráter mais repressivo (Villemor Amaral, 1978). Essa característica associada ao controle parece ser ainda mais evidente no uso da cor azul, que também traz tal conotação, com características mais racionais (Villemor-Amaral, 2012). Historicamente é atribuído aos homens um caráter mais repressor e racional do que às mulheres, consideradas mais ternas e emocionais, $\mathrm{O}$ que já foi demonstrado em estudos sobre dominância cerebral (Yazigi, 1995).

$\mathrm{Na}$ comparação entre meninas e mulheres adultas, destaca-se o fato de que as meninas tenderam a usar mais tonalidades 4 , enegrecidas tanto do azul quanto do vermelho. Talvez esse aumento nas meninas esteja associado a uma maior necessidade de contenção ou repressão das emoções e impulsos, em uma fase ainda 
de pouco amadurecimento, o que pode ser substituído por mecanismos mais adaptativos em uma faixa etária mais amadurecidade mulheres adultas.

O conjunto desses resultados sugere que a escolha das cores no TPC seguiu o padrão relatado nos estudos de LoBue e DeLoache (2011) e Milne e Greenway (1999) que investigaram as cores nos desenhos infantis, brinquedos e outros objetos refletindo a participação de questões culturais na escolha das cores. Para esses autores, essa escolha representa um estereótipo de sexo, sendo os tons mais claros e rosa, predominantemente, escolhidos por mulheres e tons mais escuros, por homens.

É interessante ressaltar que a significação das cores azul e vermelho, bem como suas tonalidades, escuras, vibrantes ou diluídas, no TPC, podem se associar a padrões culturais, na medida em que a significação delas corresponde aos padrões socialmente atribuídos a cada um dos sexos, entre tantos outros. Mas assim foram socialmente significadas em razão, muito provavelmente, de suas propriedades mais ou menos estimulantes. Desse modo, as tonalidades mais claras e suaves, que sugerem mais fragilidade, foram selecionadas mais frequentemente por mulheres $\mathrm{e}$ crianças, enquanto que as tonalidades mais escuras ou vibrantes, que denotam força ou energia, foram mais frequentes, por homens.

Esses resultados parecem confirmar uma influência mútua em que interagem tanto as propriedades físicas da cor quanto seus significados simbólicos culturais cristalizados ao longo do tempo. De qualquer modo, há indícios de que a escolha de cores difere conforme sexo e idade e, considerando que o TPC tem o objetivo de avaliar aspectos da dinâmica de personalidade (Guntert, 2000; Peres et al., 2007), nem sempre facilmente identificados no comportamento explícito do examinando (Franco, 2009), é importante estar atento para esses fatores ao interpretar o teste, tal como indicado nos estudos de Villemor-Amaral et al. (2014a), Pasian et al. (2014), Villemor-Amaral et al. (2003), Villemor-Amaral et al. (2008) e Bastos-Formighieri e Pasian (2012).

\section{Conclusão}

A presente pesquisa teve como objetivo analisar se a escolha das cores diverge conforme o sexo e a idade, sob influência de fatores culturais. O conjunto de resultados demonstra que de fato esse fenômeno ocorre em correspondência a estereótipos sociais.
Por outro lado, essa pesquisa não levou em conta dados mais específicos dos participantes, para além de idade e sexo. Em futuras investigações, a comparação de características de personalidade, avaliadas por outros instrumentos, poderão acrescentar dados valiosos para a compreensão dos significados da preferência pelas tonalidades no Pfister, para além da frequência das cores em si.

É importante destacar que diferentemente do que se pudesse precipitadamente concluir, esses resultados não devem ser tomados com um indicador de identidade de gênero ou preferências sexuais. Entretanto, pode trazer informações adicionais na compreensão do maior ou menor interesse em compartilhar de imposições culturais no caso de indivíduos com escolhas diferentes do padrão mais habitual, quando se trata do rosa e do azul. Assim como os demais estudos realizados sobre o teste de Pfister, suas contribuições ajudam a esclarecer certos aspectos do dinamismo afetivo, mas ainda são necessários estudos com amostras maiores e contextos diferentes para que se possa avançar com as evidencias de validade de uso desse instrumento.

\section{Referências}

Bastos-Formighieri, M. S., \& Pasian, S. R. (2012). O Teste de Pfister em idosos. Avaliação Psicológica, 11(3), 435-448. Recuperado de http://pepsic. bvsalud.org/scielo.php?script=sci_arttext\&pid $=$ S1677-04712012000300010

Cohen, J. (1992). A power primer. Psychological Bulletin, 112,155-159. Recuperado de http://drsmorey. org/bibtex/upload/Cohen:1992.pdf

Farah, F. H. Z., Cardoso, L. M., \& Villemor-Amaral, A. E. (2014). Precisão e validade do Pfister para avaliação de crianças. Avaliação Psicológica, 13(2), 187-194. Recuperado de http:// pepsic.bvsalud.org/scielo.php?script=sci_arttext\& pid=S1677-04712014000200006

Franco, R. R. C. (2009). Ensaio de convergência entre provas de personalidade: Zulliger-SC e Pfister (Tese de Doutorado). Universidade São Francisco, Itatiba, São Paulo, Brasil.

Guntert, A. E. V. A. (2000). Técnicas projetivas: O geral e o singular em avaliação psicológica. In F. F. Sisto, E. T. B. Sbardelini \& R. Primi (Ed.). Contextos e questões da avaliação psicológica (pp. 77-85). São Paulo: Casa do Psicólogo. 
Heller, E. (2011). Psicologia del color. Como actuanlos colores sobre lossentimientos y larazón. Editorial Gustavo Gili: Barcelona.

Hulbert, A. C., \& Ling, Y. (2007). Biological components of sex differences in color preference. Current Biology, 17, R623-R625. doi: 10.1016/j.cub.2007.06.022

LeDoux, J. (1998). The Emotional Brain. New York: Weidenfeld \& Nicolson.

LoBue, V.,\&DeLoache,J.S.(2011).Prettyinpink:Theearly development of gender-stereotyped colour preferences. British Journal of Developmental Psychology, 29, 656-667. doi: 10.1111/j.2044-835X.2011.02027.x

Marques, M. I. B. (1988). O Teste de pirâmides coloridas de Max Pfister. São Paulo: EPU - EDUC.

Milne, L. C., \& Greenway, P. (1999). Color in children's drawings: The influence of age and gender. The Arts in Psychotherapy. 26(4), 261-263. doi: 10.1016/ S0197-4556(98)00075-6

Pasian, S. R., Barroso, J. B., \& Theodozio, D. R. (2014). O Teste de Pfister na adolescência. In A. E. Villemor-Amaral. As Pirâmides de Pfister com Crianças e Adolescentes (pp. 107-126). São Paulo: Casa do Psicólogo.

Peres, R. S., Santos, M. A. Rodrigues, A. M., \& Okino, E. T. K. (2007). Técnicas projetivas no contexto hospitalar: Relato de uma experiência com o House-Tree-Person (HTP). Revista Iberoamericana de Diagnóstico e Avaliação Psicológica. 23(1), 41-62. Recuperado de http://www.aidep.org/03_ridep/R23/ R233.pdf

Silva, L. M., \& Cardoso, L. M. (2012). Revisão de pesquisas brasileiras sobre o teste de pfister. Avaliação Psicológica, 11(3), 449-460. Recuperado de http://pepsic.bvsalud.org/scielo.php?pid=S1677-04712012000300011\&script=sci_arttext

Villemor Amaral, F. (1978). Pirâmides Coloridas de Pfister. $2^{\mathrm{a}}$ ed. Rio de Janeiro: CEPA.

Villemor-Amaral, A. E. (2012). O teste das pirâmides coloridas de Pfister. São Paulo: Casa do Psicólogo.

Villemor-Amaral, A. E. (2014). As pirâmides de Pfister com crianças e adolescentes. São Paulo: Casa do Psicólogo.

Villemor-Amaral, A. E., \& Quirino, G. S. (2013). Estudo comparativo entre indicadores afetivos das técnicas de Pfister e Zulliger. Avaliação psicológica, 12(1), 1-7. Recuperado de http://
pepsic.bvsalud.org/scielo.php?pid=S1677$-04712013000100002 \&$ script $=$ sci_arttext

Villemor-Amaral, A.E.Pardini,P.M., Tavella, R. R., Biasi, F. C., \& Migoranci, P. B. (2012). Evidências de validade do teste de Pfister para avaliação de crianças. Avaliação psicológica, 11(3), 423-434. Recuperado de http://pepsic.bvsalud.org/scielo.php?pid=S1677$-04712012000300009 \&$ script $=$ sci_arttext

Villemor-Amaral, A. E., Farah, F. H. Z., \& Primi, R. (2004). O teste das pirâmides coloridas e o transtorno do pânico. Psicologia Estudos, Maringá, 9(2), 301-307. doi: 10.1590/S1413-73722004000200016

Villemor-Amaral, A. E., Pianowski, G., \& Gonçalves, C. M.T.S.(2008).EstudonormativocomoPfister:Uma amostra da região nordeste brasileira. Avaliação Psicológica, Porto Alegre, 7(2), 181-188. Recuperado de http:// pepsic.bvsalud.org/scielo.php?pid=S1677$-04712008000200009 \&$ script $=$ sci_arttext

Villemor-Amaral, A. E., Primi, R., Farah, F. H. Z., Cardoso, L. M., \& Franco, R. R. C. (2003). Revisão das expectativas no Pfister para uma amostra normativa. Avaliação Psicológica, 2(2), 185-188. Recuperado de http://pepsic.bvsalud.org/scielo.php?pid=S1677-04712003000200009\&script $=$ sci_arttext

Villemor-Amaral, A. E., Primi, R., Farah, F. H. Z., Silva, S. M., Cardoso, L. M., \& Franco, R. R. C. (2004). A depressão no teste das pirâmides coloridas de Pfister. Paidéia: Cadernos de psicologia e educação, 14(28), 169-179. Recuperado de http://www.scielo.br/ pdf/paideia/v14n28/06.pdf

Villemor-Amaral, A. E., Primi, R., Franco, R. R. C., Farah, F. H. Z, Cardoso, L. M., \& Silva, T. C. (2005). O teste de Pfister e sua contribuição para o diagnóstico da esquizofrenia. Revista do Departamento de Psicologia, Universidade Federal Fluminense, Niterói, 17(2), 89-98. doi: 10.1590/ S0104-80232005000200008

Villemor-Amaral, A. E., Silva, T. C., \& Primi, R. (2002). O teste das pirâmides coloridas de Pfister e o transtorno obsessivo compulsivo. Avaliação Psicológica, Porto Alegre. 1(2), 133-139. Recuperado de http:// pepsic.bvsalud.org/scielo.php?script $=$ sci_arttext\& pid $=$ S1677-04712002000200006

Villemor-Amaral, A. E., Silva, T. C., \& Primi, R. (2003). Indicadores de alcoolismo no teste das pirâmides coloridas de Pfister. Psico- USF, Itatiba, 8(1), 33-38. 
Recuperado de http://www.scielo.br/pdf/pusf/ v8n1/v8n1a05.pdf

Villemor-Amaral, A. E., Cardoso, L. M., Pavan, P. M. P., Tavella, R. R., Biasi, F. C., \& Miguel, F. K. (2014a). Estudos psicométricos. In A. E. Villemor-Amaral. As pirâmides coloridas de Pfister: Versão para crianças e adolescentes (pp.53-106). São Paulo: Casa do Psicólogo.

Villemor-Amaral, A. E., Cardoso, L. M., Pavan, P. M. P., Tavella, R. R., \& Biasi, F. C. (2014b). Validade e precisão: Estudos Recentes. In A. E.
Villemor-Amaral. As pirâmides coloridas de Pfister: Versão para crianças e adolescentes (pp.127-147). São Paulo: Casa do Psicólogo.

Yazigi, L. (1995) A prova de Rorschach, a especialização hemisférica e a epilepsia. Livre Docência/Unifesp-EPM/São Paulo.

Recebido: 22/06/2014

Primeira reformulação: 23/12/2014

Aprovado: 19/03/2015

Nota das autoras:

Agradecimento: $\mathrm{CNPq}$

Sobre as autoras:

Anna Elisa de Villemor-Amaral é doutora em Ciências pela Unifesp/EPM e docente do Programa de Pós-Graduação Stricto Sensu em Psicologia da Universidade São Francisco - Campus Itatiba.

E-mail: anna.villemor@saofrancisco.edu.br

Fabiola Cristina Biasi é mestre em Psicologia na Universidade São Francisco e docente do curso de graduação em Psicologia na mesma universidade - Campus Campinas e Itatiba.

E-mail: fabiola.biasi@usf.edu.br

Lucila Moraes Cardoso é doutora em Psicologia pela Universidade São Francisco e docente no curso de graduação em Psicologia da Universidade Estadual do Ceará (UECE).

E-mail: lucilamcardoso@yahoo.com.br

Pâmela Malio Pardini Pavan é mestre pelo Programa de Pós-Graduação Stricto Sensu em Psicologia da Universidade São Francisco - Campus Itatiba.

E-mail:papavan@gmail.com

Raquel Rossi Tavella é mestre em Psicologia pela Universidade São Francisco e docente no curso de graduação em Psicologia do Centro Universitário Nossa Senhora do Patrocínio (CEUNSP) - Campus Itu.

E-mail: raqueltavella@yahoo.com.br.

Contato com as autoras:

Anna Elisa de Villemor-Amaral

E-mail: anna.villemor@saofrancisco.edu.br

Rua Alexandre Rodrigues Barbosa, 45, Centro, Itatiba - SP

CEP: $13251-900$ 